\title{
Blocking L-Type Calcium Current Reduces Vulnerability to Re-Entry in Human iPSC-Derived Cardiomyocytes Tissue
}

\author{
Albert Dasi ${ }^{1}$, Andreu M Climent ${ }^{2}$, Laura Martinez ${ }^{1}$, Juan F Gomez ${ }^{1}$, Jose M Ferrero ${ }^{1}$, Beatriz Trenor ${ }^{1}$ \\ ${ }^{1}$ Universitat Politècnica de València, Valencia, Spain \\ ${ }^{2}$ Instituto de Investigación Sanitaria Gregorio Marañón, Madrid, Spain
}

\begin{abstract}
Human induced pluripotent stem cell-derived cardiomyocytes (hiPSC-CMs) have proven to be crucial in pharmacological assessment. Nevertheless, their response to drugs when coupled forming a tissue is not fully understood. Thus, the aim of this study was to determine whether blocking L-type $\mathrm{Ca}^{+2}$ current $\left(\mathrm{I}_{\mathrm{CaL}}\right)$ in a hiPSCCMs tissue could be considered as a potential antiarrhythmic procedure.

To analyze the effects of $I_{C a L}$ block, the maximum conductance of $I_{\text {CaL }}\left(g_{\text {CaL }}\right)$ was decreased (block conditions) and compared to control. In both situations, control and block, the tissue was stimulated following a cross-field protocol to generate re-entries. A phase analysis was performed and specific parameters, such as re-entry frequency $\left(f_{\text {reentry }}\right)$, excitation wavelength, vulnerable window $(V W)$, and cellular excitability, were evaluated.

Induced re-entries, where $I_{\text {CaL }}$ was reduced by $70 \%$ showed $a \quad 6.9 \%$ and a $47.83 \%$ decrease in $f_{\text {reentry }}$ and in the width of the $V W$, respectively. Our results suggest that blocking calcium channels could be considered as an antiarrhythmic strategy in a hiPSC-CMs tissue.
\end{abstract}

\section{Introduction}

Newer and more sensitive techniques of cell maturation have allowed the development of human induced pluripotent stem cell derived cardiomyocytes (hiPSC$\mathrm{CMs}$ ) and their use to predict the electrophysiological behavior of adult cardiomyocytes (CMs). As a result, hiPSC-CMs are taking a growing role in cardiac research.

Specifically, in pharmacological assessment, the combined in vitro and in silico approach set out by the CiPA [1] initiative, pointed out the interest of using hiPSCCMs in cardiotoxicity tests. Accordingly, this technology represents an optimal scenario to study the arrhythmogenic effects of specific drugs.

Different studies have analyzed in silico the effects of ion currents modification on the action potential (AP) of a hiPSC-CM model [2], highlighting a high sensitivity to L- type $\mathrm{Ca}^{+2}$ current $\left(I_{C a L}\right)$ block. Therefore, the extension of this research considering AP propagation when cells are coupled is promising.

In this work, we analyze the establishment and nature of re-entries in a virtual hiPSC-CMs tissue and evaluate the effects of blocking $I_{C a L}$. $I_{C a L}$ reduction can be achieved in vitro by administrating different selective ion channel blockers such as verapamil.

\section{Methods}

To analyze the arrhythmogenic behavior under the effects of $I_{C a L}$ block in hiPSC-CMs, a two-dimensional tissue was modeled using the latest version of the AP model for hiPSC-CM [3]. Material conditions, such as conduction velocity (CV) and the level of anisotropy, were defined on the basis of experimental data [4].

In order to identify the effect of blocking $I_{C a L}$, two different situations were studied: one established as control, with no changes in the model parameters, and another where the maximum conductance of $I_{C a L}\left(g_{C a L}\right)$ was decreased by $70 \%$ (block).

Arrhythmogenicity was evaluated using several biomarkers, such as the excitation wavelength, re-entrant frequency $\left(f_{\text {reentry }}\right)$, vulnerable window (VW) for re-entry and cellular excitability. Furthermore, we performed a phase analysis where the phase singularity (PS) points were detected to track the movement of the rotor tip [5-7].

\subsection{Vulnerable window for re-entry}

Re-entries were induced following a cross-field stimulation protocol S1-S2. The critical time interval, within which stimulus $\mathrm{S} 2$ was applied and generated a reentrant rhythm was determined in both situations, control and block, to characterize the VW for re-entry (beginning, duration and end). Once the VWs were obtained in control and block, a detailed analysis of a re-entry was analyzed for both situations. To assure equivalent conditions of the compared re-entries, in both cases the re-entry generated after 45 milliseconds (ms) of the lower limit of the VW was selected. 


\subsection{Phase map analysis}

The wavelength of excitation was mainly quantified through the perimeter of the rotor tip. The detection of the PS points enabled the generation of binary files, establishing the PS points as hyperintense and the rest of the tissue as hypointense. These files were processed using morphological image processing to calculate the perimeter described by the PS points. The phase map analysis was carried out applying the Hilbert Transform (HT) to the membrane potential $\left(V_{m}\right)$ (Equation 1), leaving $500 \mathrm{~ms}$ from the beginning and end of the simulation, so that transformation errors could be avoided. Thus, we obtained a phase vector (Equation 2) for each point of the tissue that ranged between $-\pi$ and $\pi$. PS points were established as the points where all phases converged in each temporary instant.

$$
\begin{gathered}
H T\left[V_{m}(t)\right]=\frac{1}{\pi} \int_{-\infty}^{\infty} \frac{V_{m}(\tau)}{t-\tau} d \tau \\
\theta=\tan ^{-1}\left(\frac{H T\left[V_{m}(t)\right]}{V_{m}(t)}\right)
\end{gathered}
$$

\subsection{Frequency of the re-entry}

The $f_{\text {reentry }}$ was calculated as the average of the excitation frequency of every single point of the tissue. In each point, the frequency was computed as the inverse of the basic cycle length (BCL). Likewise, considering that the simulation was long enough to nest several re-entry loops, the BCL was calculated as the average of the BCLs of each loop.

\subsection{Cellular excitability}

Cellular excitability was evaluated through the analysis of the main currents involved in the cellular depolarization, i.e. fast $\mathrm{Na}^{+}$current $\left(I_{\mathrm{Na}}\right)$ and $I_{\mathrm{CaL}}$, as well as the product of the $I_{N a}$ inactivation gates " $h$ " and " $j$ ". The value and time course of these parameters were compared in an excitable and a non-excitable node of control and block virtual tissues. In this way, we looked for the existence of specific nodes where the excitation wavefront was propagated in control and extinguished in block conditions due to the lack of excitability. Additionally, we analyzed the variables of one point in the block conditions where the wavefront was propagated, to observe the propagation requirements, and one point from control tissue where the wavefront was extinguished.

\section{Results}

The substantial $I_{C a L}$ reduction leads to a greater dependency of $I_{N a}$ during cellular depolarization. Accordingly, only those cells showing high $I_{N a}$, and thus higher availability of inactivation gates " $h$ " and " $j "$, will be able to develop an AP. As a result, cells under block conditions have lower propensity to be excited, so that the wavefront meanders in the tissue trying to propagate in available regions. Therefore, an increase of the meandering in the rotor tip movement (Fig. 1) can be observed in the phase maps.

In the control tissue, the tip is located in a small zone, whereas in block conditions each loop exhibits a bigger circuit (Fig. 2). In the first re-entry loop, the distance travelled by the rotor tip was $0.115 \mathrm{~cm}$ during $386 \mathrm{~ms}$ in

\section{CONTROL}
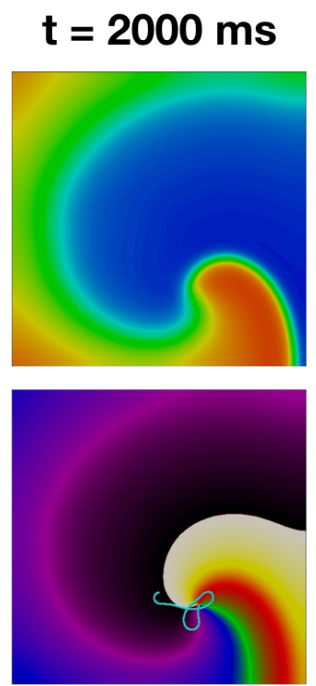
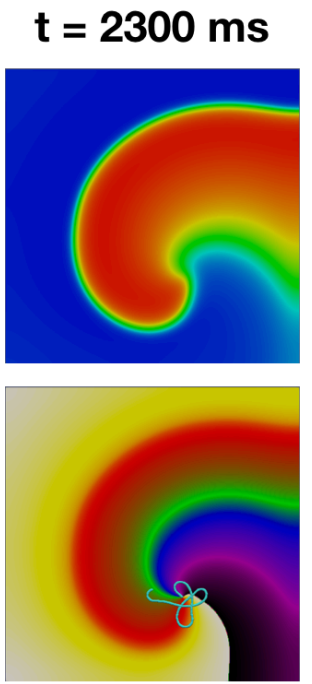

\section{BLOCK}

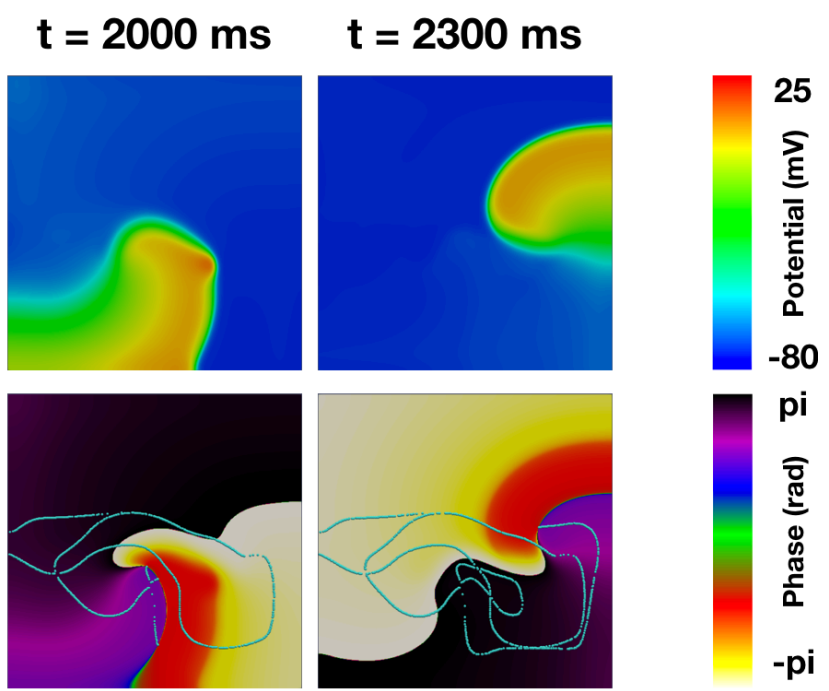

Figure 1. Membrane potential and phase maps snapshots at different instants of time. Comparison of the rotor tip movement - represented by the blue line drawn in the phase maps - between control and block conditions. The timing reference above each snapshot corresponds to the elapsed time after the S1 stimulus. 
control tissue, whereas in block conditions the distance increased to $1.212 \mathrm{~cm}$ and it took $724 \mathrm{~ms}$.

\section{CONTROL}

$\mathrm{t}=1635 \mathrm{~ms}$

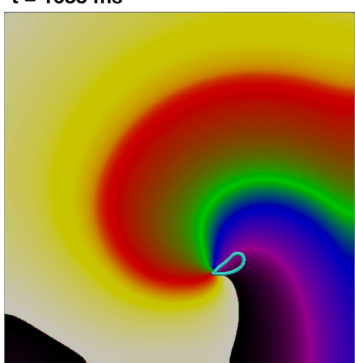

BLOCK

$\mathrm{t}=1724 \mathrm{~ms}$

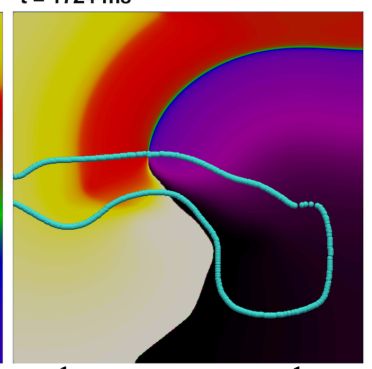

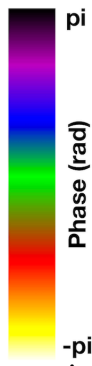

Figure 2. Instantaneous phase maps and rotor tip movement in control (left) and block (right) conditions. The instants captured were after S1 stimulus.

Figure 3A illustrates the location of the points where propagation succeeds (blue and green) or not (purple and yellow). Figure 3B shows the time course of the membrane potential, the $h$ and $j$ product, and $I_{N a}$ and $I_{C a L}$ measured in those nodes. In the control tissue a great contribution of $I_{C a L}$ in cellular depolarization can be observed, having a higher amplitude than $I_{N a}$ (blue). Under block conditions, when the wavefront makes a complete rotation and reaches those cells which have been recently stimulated (yellow), the product of the inactivation gates " $h$ " and " $j$ " is not high enough to produce a significant $I_{N a}$ that can compensate the calcium depression (comparison between yellow and green $I_{N a}$ and $h * j$ curves). Thus, the maximum potential reached (yellow) is not big enough to propagate. Consequently, the extinction of the AP occurs, and the wavefront moves towards more excitable regions. The unique non-excitable regions in the control tissue are those where the stimulus arrives within the refractory period of the cells in these regions, when $h * j$ is very low and $I_{N a}$ is not activated (purple).

The movement of the wavefront in block conditions favors an increase of the excitation wavelength and proves the reduction of cellular excitability in block conditions. Considering that $f_{\text {reentry }}$ is inversely proportional to the excitation wavelength, an important increase of the wavelength (Fig. 2) leads to the reduction of the $f_{\text {reentry }}$, as shown in Fig. 4. Additionally, the decrease in excitability has reduced the width of the VW by $47.83 \%$ from $115 \pm 5 \mathrm{~ms}$ to $60 \pm 5 \mathrm{~ms}$ (Table 1 ).

Table 1. VW characteristics in control and block conditions for the hiPSC-CMs tissue.

\begin{tabular}{ccc}
\hline Situation & Interval $(\mathrm{ms})$ & WV $(\mathrm{ms})$ \\
\hline Control & {$[475,480]-[590,595]$} & $115 \pm 5$ \\
Block & {$[550,555]-[610,615]$} & $60 \pm 5$ \\
\hline
\end{tabular}

A
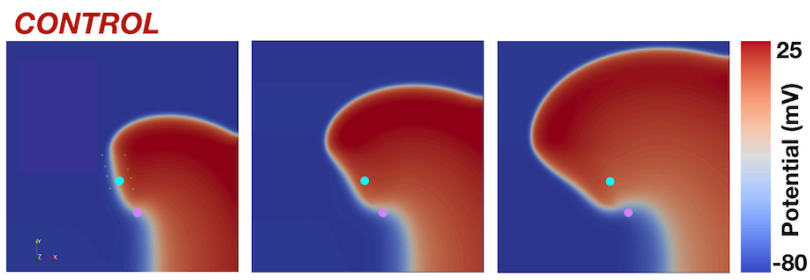

BLOCK
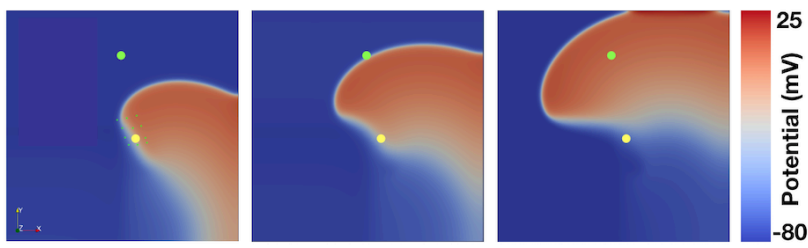

B
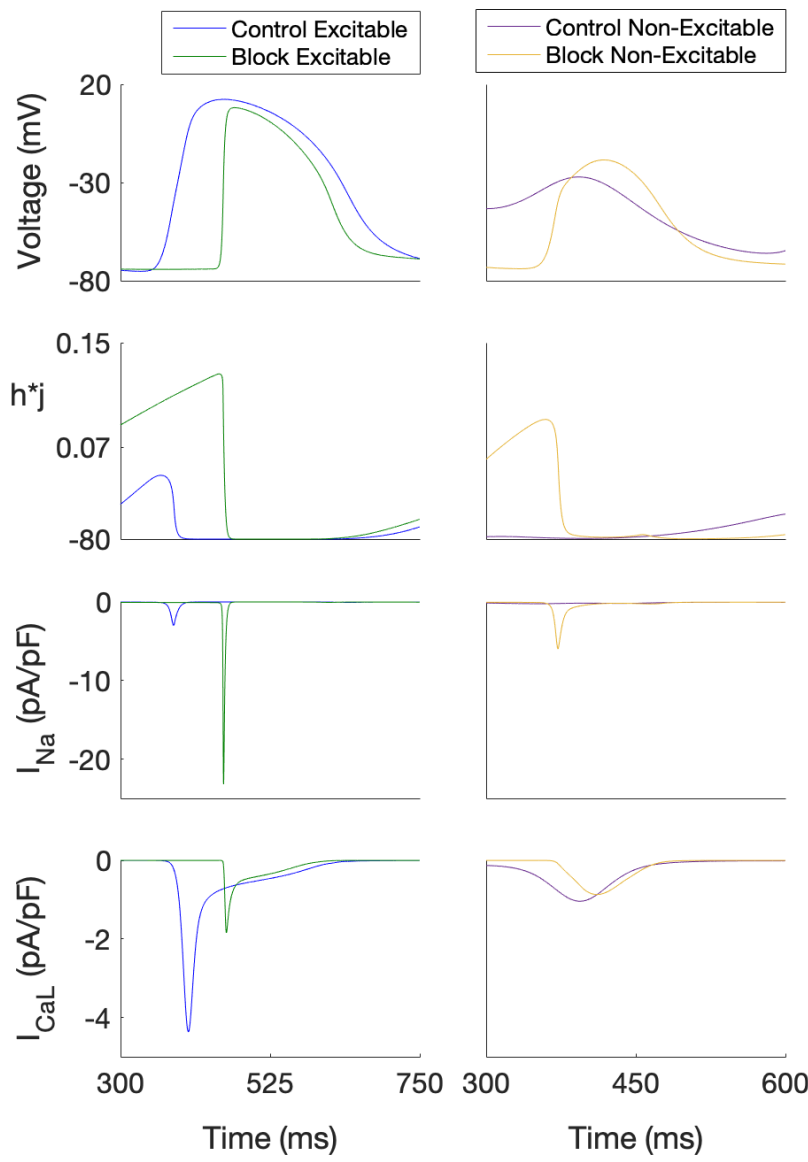

Figure 3. Analysis of cellular excitability. A) Location of the tissue nodes studied in each situation, control and block. B) Comparison of the membrane potential (Voltage), sodium current $\left(I_{N a}\right)$, calcium current $\left(I_{C a L}\right)$, and the product of the sodium channel inactivation gates " $h$ " and " $j "(h * j)$ in those nodes. 


\section{Discussion and conclusions}

Our results suggest that blocking calcium channels could be considered as an antiarrhythmic strategy in a hiPSC-CMs tissue, since meandering of the rotor tip promotes a reduction in the $f_{\text {reentry }}$ and the tissue excitability reduction produces a decrease in the VW width. Therefore, besides establishing a re-entry which tends to vanish due to all the meandering needed to find excitable regions, it has less probability to appear in the first place.

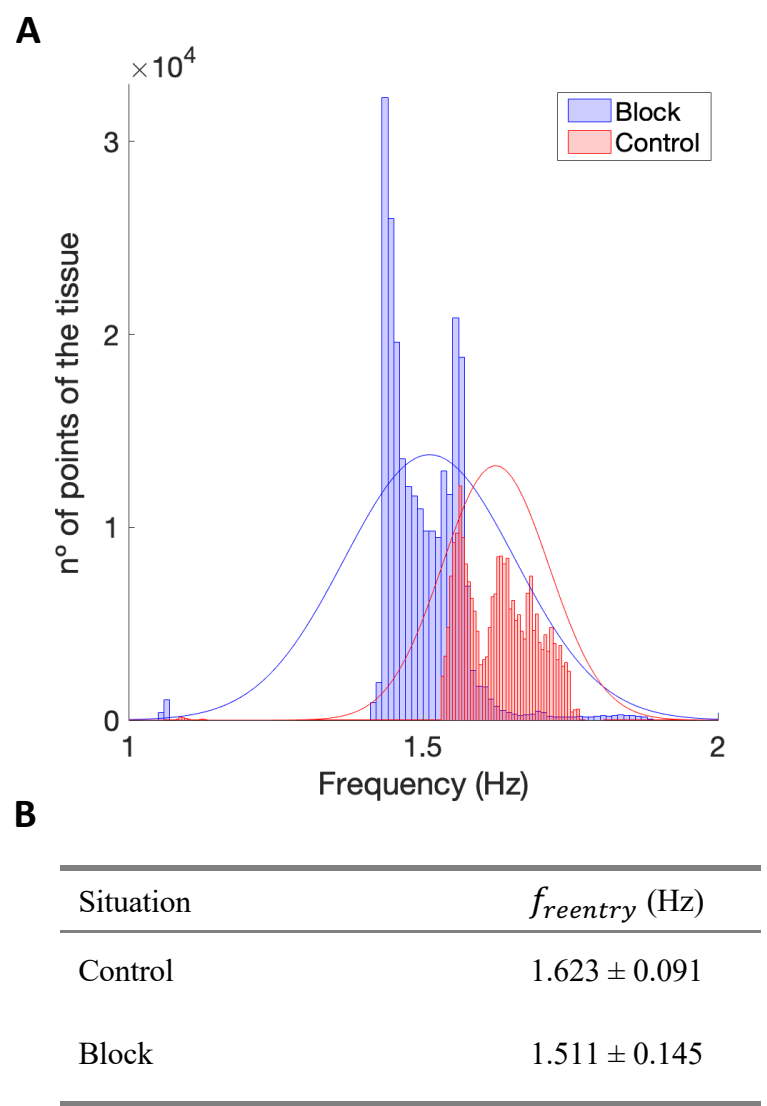

Figure 4. A) Histogram of the excitation frequency in all the nodes of the tissue. B) Mean value of the excitation frequency in both situations, control and block.

Although there are a lot of experiments analyzing the effects of blocking $I_{C a L}$ in different cardiac tissues, this procedure is still being controversial. Some studies [8] state that the reduction of the AP duration (APD), related to $I_{C a L}$ depression, should be considered as proarrhythmic because it might produce an acceleration of the re-entrant rhythm. This work proves that, far from this remark, $I_{C a L}$ reduction not only decreases the frequency of the re-entry but it also hampers the re-entry establishment in a hiPSCCMs tissue.

\section{References}

[1]. T. Colatsky, B. Fermini, G. Gintant et al., "The Comprehensive in Vitro Proarrhythmia Assay (CiPA) initiative - Update on progress", $V$. J Pharmacol Toxicol Methods, vol. 81, pp. 15-20, Sep.-Oct. 2016.

[2]. M. Paci, J. Hyttinen, B. Rodriguez et al., "Human induced pluripotent stem cell-derived versus adult cardiomyocytes: An in silico electrophysiological study on effects of ionic current block", British Journal of Pharmacology, vol. 172, no. 21, pp. 5147-5160, Aug. 2015.

[3]. M. Paci, R. P. Pölönen, D. Cori et al., "Automatic optimization of an in silico model of human iPSC derived cardiomyocytes recapitulating calcium handling abnormalities", Frontiers in Physiology, vol. 9, pp. 1-14, Jun. 2018.

[4]. E. Garreta, L. de Oñate, M. Fernández-Santos et al., "Myocardial commitment from human pluripotent stem cells: Rapid production of human heart grafts", Biomaterials, vol. 98, pp. 64-78, Aug. 2016

[5]. L. Martínez-Mateu, L. Romero, A. Ferrer-Albero et al., "Factors affecting basket catheter detection of real and phantom rotors in the atria: A computational study", PLOS Computational Biology, vol. 14, no. 3, pp. 1-26, Mar. 2018.

[6]. R. Gray, A. Pertsov, J. Jalife, "Spatial and temporal organization during cardiac fibrillation", Nature, vol. 392, no. 6671 , pp. 75-78, May. 1998.

[7]. J. Gomez, K. Cardona, L. Romero et al., "Electrophysiological and Structural Remodeling in Heart Failure Modulate Arrhythmogenesis. 2D Simulation Study", PLoS ONE, vol. 9, no. 9, Jul. 2014.

[8]. F. Chorro, J. Cánoves, J. Guerrero et al., "Alteration of ventricular fibrillation by flecainide, verapamil, and sotalol: an experimental study", Circulation, vol. 101, no. 13, pp. 1606-1615, Nov. 1999.

Address for correspondence:

Albert Dasí

Ci2B, Universitat Politècnica de València

Camino de Vera s/n, 46022 Valencia, Spain

aldaim@etsii.upv.es 